Health \& Medicine | Claire Kendal-Wright

\section{Preterm birth and danger-associated molecular patterns}

Preterm birth is the leading
cause of infantile mortality.
Dr Claire Kendal-Wright from
the Chaminade University of
Honolulu works on human extra-
embryonic membranes. More
specifically, she studies danger-
associated molecular patterns,
or DAMPs, and examines how
these molecules contribute to
the prelabour rupture of the
membranes that leads to birth.
Understanding the mechanisms
involved in healthy pregnancy
could eventually allow the
development of therapies
aiming at preventing
preterm birth.

Every year,woldwide, an estimated United States, much of the impact of - 15 million babies are born preterm birth is borne by ethnic minority babies While a tormat pegan 1 in 10 babies. While a nomal pregnancy lasts to be preterm or premature when occurs before 37 weeks of pregnancy are completed. The earlier a baby is born, the higher the risk of death or serious disability. Preterm birth is one of the most common negative pregnancy outcomes as the resulting complications are the leading cause of death among children under 5 years of age.

Rates of preterm birth vary across countries and range from $5 \%$ to $18 \%$ of babies born. The incidence of pretern

Fetal Membranes

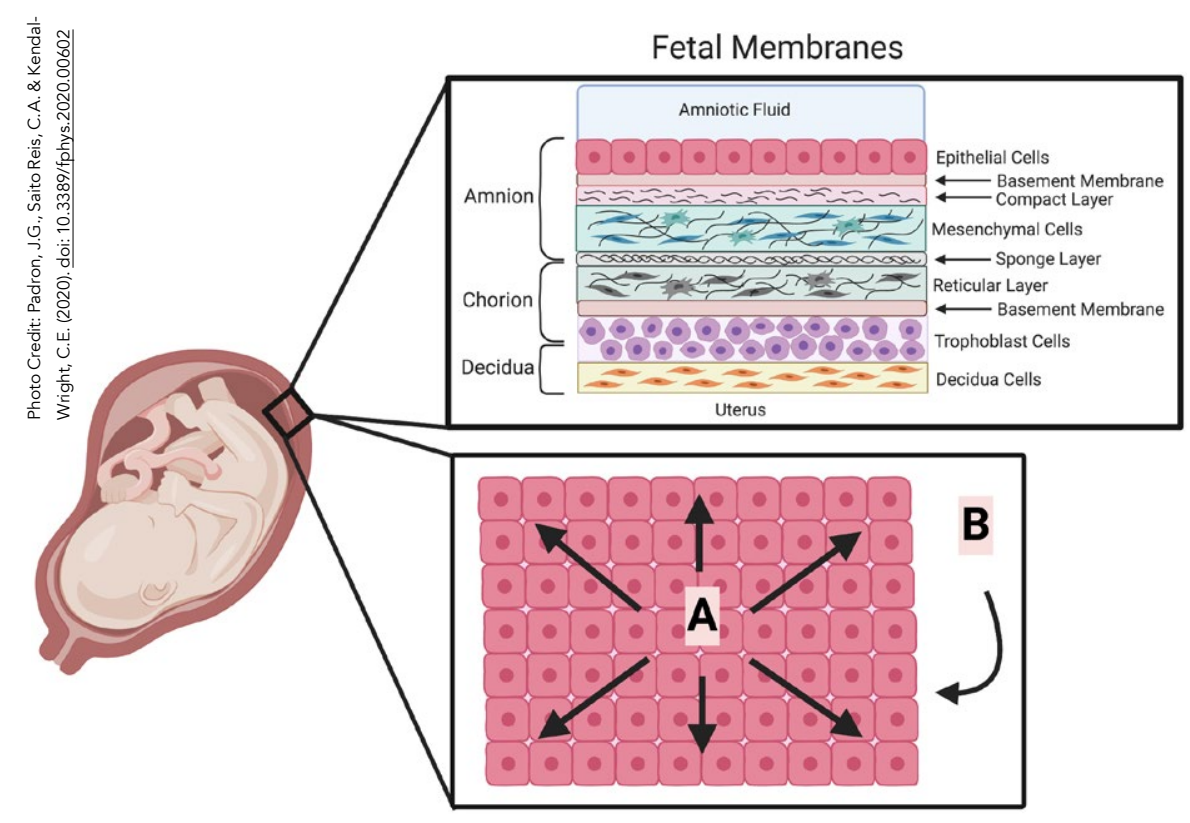

Fetal membranes consist of a multi-layered structure (upper box). Biophysical forces are placed
on these membranes (lower box): linear physical forces due to multi-directional stretching of the membranes adherent to the decidua (A) and
Braxton Hicks contractions and labour $(B)$.
Cire Kendal-Wright, who works at the Chaminade University research on premary tocuses her the Pacific Islands, where preterm birth is an issue mostly for the native peoples. Indeed, the infant mortality rate is twice as high for Native Hawaiian mothers compared to Caucasians, and almost half of this infant mortality is related to preterm birth.

\section{PHYSIOLOGICAL CHANGES} LEADING TO BIRTH

As pregnancy comes to term, different physiological changes lead to birth: the cervix softens and dilases to allow the passage of the foetus, the myometrium (muscular layer of the uterine wall) contracts, the placenta separates breaking". These distinct physiological events need to occur in a coordinated manner for the successful delivery of the oetus at term. Indeed, desynchrony or dysregulation of these events can lead to preterm birth

Preterm birth can result from different events. Approximately $20 \%$ of all preterm deliveries are by Caesarean section for maternal or foetal indications. Of the remaining premature prelabour rupture of the the remainder are duetion, and uterine contractions. the foetal mem, and the rapture of cases, around $30-35 \%$ are caused by membranes (PPROM), 20-25\% result
The trigger that initiates the physiological changes leading to birth $60 \%$ of preterm biths, the reason delivery occurred eal ier than expected is unknown. Understanding what

happens in normal pregnancy and how abour onset is triggered is important because it can help explain how the physiological mechanisms may deviate and lead to preterm birth.

OETAL MEMBRANES

The foetal membranes (the amnion and the chorion) surround the developing embryo and form an interface between the foetus and the mother. The amnion is the innermost layer. It is in contact with the amniotic fluid, the foetus wid the un

Amnion and chorion maintain the conditions necessary for foetal health and therefore protect the foetus during pregnancy. As pregnancy advances to term, the foetal membranes undergo rupture. The first step in the weakening is the separation of amnion and chorion which, during pregnancy, are in contact. Then, as a result of both physical forces and biochemical changes, the membranes can break. Rupture of the membranes usually triggers labour and delivery; that is when we talk about prelabour rupture of the membranes (PROM). The timing of PROM is crucia for healthy pregnancy outcomes. If ( bith. Foetal membranes and PPROM are the main focus of Dr KendalWright's work.

THE ROLE OF CELLULAR STRESS There is more and more evidence suggesting that cellular stress is the trigger for both foetal membrane occurs wh and labour. Cellular stress damaging environmental conditions. In return, cellular protection mechanism are set into motion. One of these mechanisms is inllammation, which been shown to occur during labour.

Distension of the foetal membranes, which stretch as the foetus grows, is

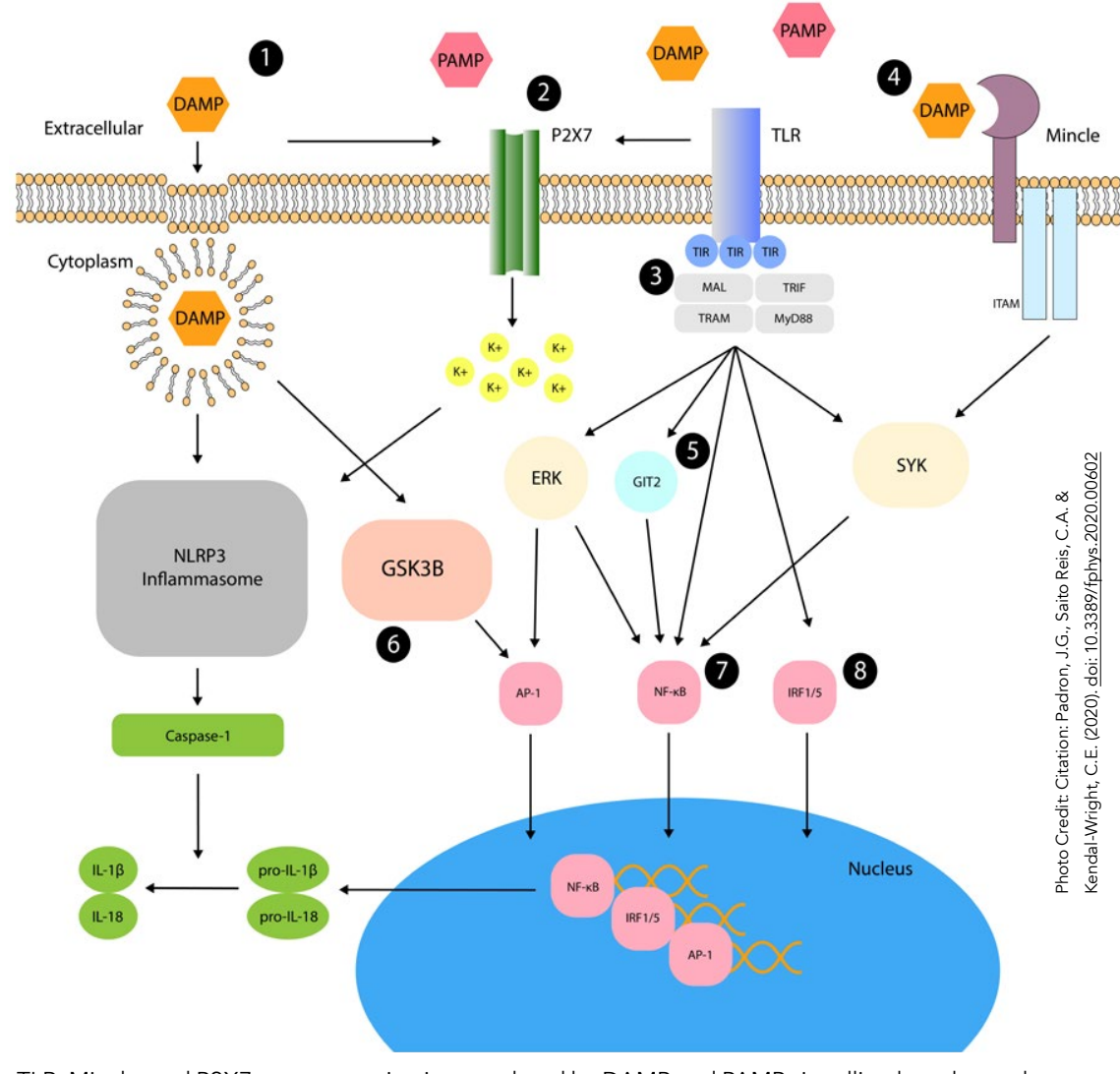

TLR, Mincle, and P2X7 receptor activation regulated by DAMP and PAMP signalling have been shown
to stimulate downstream pathways involving various modulatory proteins. This may activate AP-1, to stimulate downstream pathways involving various modulatory proteins. This may activate AP-1,
$\mathrm{NFKB}, \mathrm{R} F 1 / 5$, or inflammasome activation that leads to induction of pro-inflammatory cytokines.

membrane weakening and labour. Oxidative stress is another potentia stressor: as cells of the amnion age harmful molecules accumulate and damage the cells. Both distension of the foetal membranes and dxidative stress are known to stimulate inflammation.

FROM CELLULAR STRESS TO PROM

The production of proinflammatory (firtions first increases the production of DAMPs. Then, DAMPs interact with molecules named Toll-like receptors (ILRs), which activate the transcription factor NF-kB. As a transcription factor, $\mathrm{NF}-\mathrm{kB}$ can modul gene expression and In response to tissue injury, damaged production of proinflammatory cytokines.

There is more and more evidence suggesting that cellular stress is the trigger for both foetal membrane weakening and labour.

molecules named danger-associated molecular patterns (DAMPs). It is through DAMPs that distension and oxidative stress could stimulate inflammation and weaken the foeta membranes. Indeed, the general raise the function or DAMPs is to raise the alarm within tissues, which they do by producing a number cytokines promote cellular changes. Within the foetal membranes,

components of the extracellular matrix are

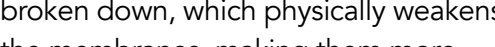
the membranes, mane to rupture.
prons

\section{DAMPS IN PRETERM BIRTH} cytokines, inflammation and rupture
Cellular stress, DAMPs, proinflammatory 


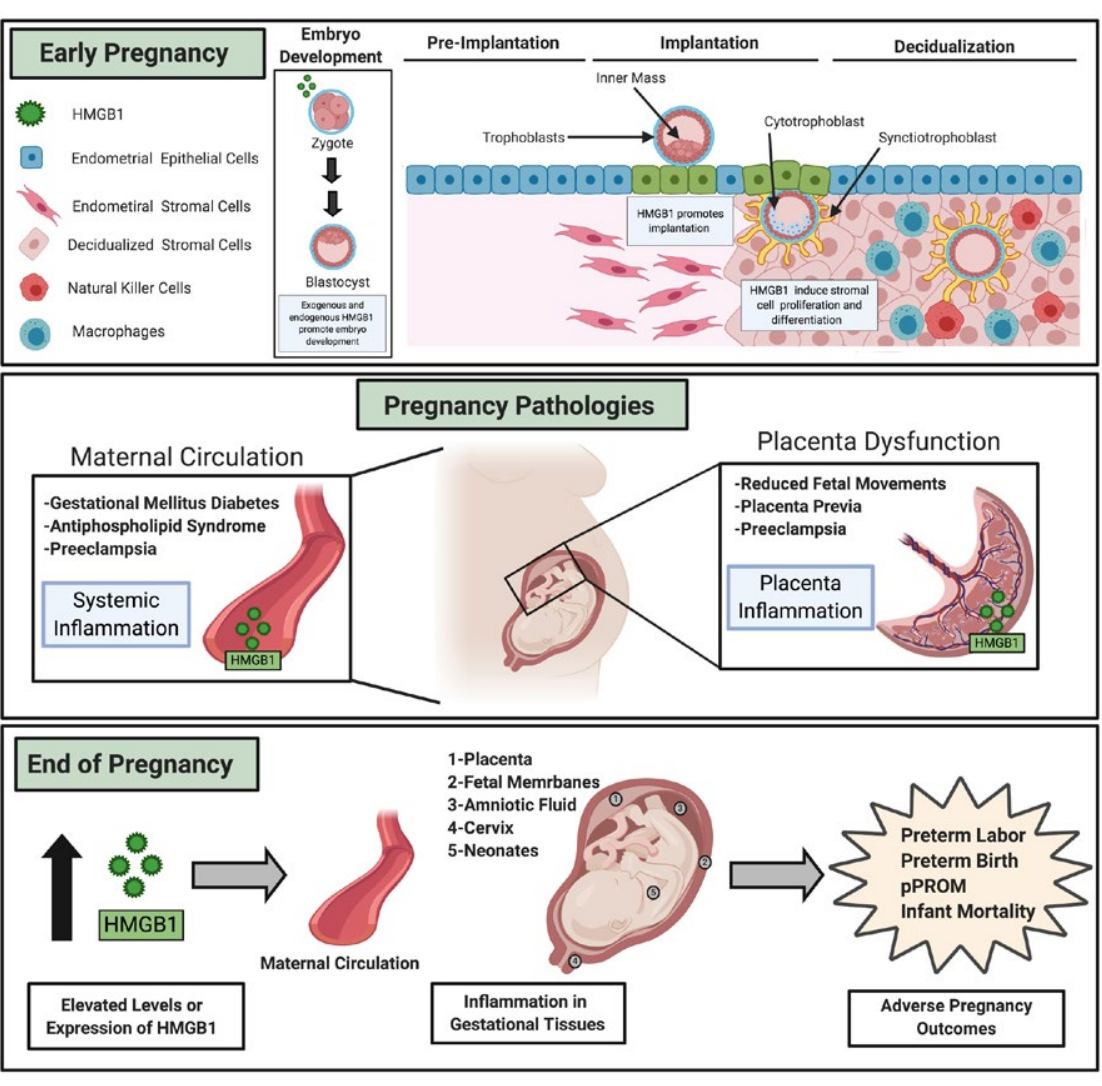

The inflammatory role of HMGB1 throughout pregnancy.

Within the foetal membranes, components of the extracellular matrix are broken down, which weakens the membranes and makes them more likely to rupture.

of the foetal membranes are involved in healthy pregnancy. However, while this cascade of events normally occurs triggered prematurely, and PROM an then cause preterm birth.

In preterm birth with $\mathrm{PPROM}$, there is evidence that DAMP-driven inflammation is associated with foetal membrane weakening. Levels of DAMPs are higher in foetal membranes from women who had preterm labour or pPROM, and this can be due to different causes. Inflammation initiated by bacterial infection causes premature birth by preterm labour and PPROM. The initiation of this inflammation is mediated by pathogen-associated molecular patterns (PAMPs), which are very similar to DAMPs but originate dying cells, Like DAMPs, PAMPs inter with TLRs and promote inflammation. Likewise, viral infection elicits inflammation through the activation of TLRs.

While bacterial or viral infections can be involved, they are not necessary: inflammation in the absence of infection (called sterile inflammation), which likely occurs due to cellular stress, can also lead to pPROM. Different causes can thus increase levels of DAMPs, and higher levels are related to higher risks of preterm birth.

\section{AN EXAMPLE OF DAMP}

One DAMP, named high mobility group box 1, Or HMGB1, has been particularly studied. Its levels are elevated in membrane extracts from preterm labour compared to term labour. HMGBI can or secreted in response to infection.
It increases levels of proinflammatory cytokines and is likely to pr

MGB1 has been shown to play a critical role not only for labour onset pregnancy Indeed, a proinflammatoy response driven by HMGB1 is essential for the development and implantation of the embryo. HMGB1 evels then need to be tightly regulated throughout pregnancy as high levels within the uterine cavity have been linked to pregnancy failure. In addition, chronic inflammation, resulting from increased HMGBI within the maternal circulation and gestational tissues, also

\section{AELICATE BALANCE}

The inflammatory response mediated Dy DAMPs such as HMGB1 in the early but excessive DAMPs levels within the uterine cavity and expression within the placenta have been shown to lead to pregnancy loss.

Once the embryo is implanted, an anti-inflammatory environment is key for the maintenance of pregnancy up until the initiation of labour. Thus, during the greater part of gestation, elevated DAMPs levels can promote the inappropriate proinflammatory signalling that is central to many preeclampsia, gestational diabetes and preeclampsia, gestational

Finally, at the end of pregnancy, inflammation is needed for the normal process of birth. But once again the balance is delicate since excessively high levels of DAMPs could initiate delivery too early and lead to preterm birth or infant mortality.

\section{DAMPS AS THERAPEUTIC TARGETS} Due to the link between DAMPs and several pregnancy-related pathologies, the possibility of use DAMPs such as HMGB 1 as a biomarker has been assessed. However, further research is needed to examine whether HMGB1 whibitors could reduce inflammation within pregnancy, so that preterm birth
could be avoided.

\section{Behind the Research}

\section{Dr Claire Kendal-Wright}

W: claire.wright@chaminade.edu T: $+8087398343 \quad$ W: $\underline{\text { https: } / / \text { chaminade.edul }}$

Research Objectives

Dr Kendal-Wright studies Danger Associated Molecular Patterns (DAMPs) and their link to infection-driven preterm birth.

Detail

Claire Kendal-Wright

Natural Sciences and Mathematics

Chaminade University of Honolulu

3140 Waialae Ave

Claire Kendal-Wright is a first-generation scientist, born and raised in the UK. Early in her career, she was fascinated by neuroscience; however, Dr Kendal-Wright pivoted her research focus to address the high rates peorm birth when she realised how impactful this iss
to the people of Hawaili and their future health.

Funding

Foundatio

National Institutes of Health (NICHD and NIGMS)

\section{Collaborators}

Dr Chelsea Sato Reis

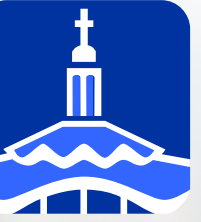

Chaminade

University

\section{References}

Padron, J. G., Saito Reis, C. A., \& Kendal-Wright, C. E. (2020). The Role of Danger Associated Molecular Pattern physiology, 11, 602. Available at: https://doi.org/10.3389/ fphys.2020.00602

Saito Reis, C. A., Padron, J. G., Norman Ing, N. D. \& Kendal-Wright C. E. (2020). High-mobility group box 1 is driver of if, C. E. (2020). High-mobilly group box 1 is journal of reproductive immunology (New York, N.Y. 1989), 13328. Advance online publication. Available at: https://doi.org/10.1111/aji.13328

World Health Organization (2018). Preterm birth. [online] World Health Organization. Available at: https:/ /www who. int/news-room/tact-sheets/detail/preterm-birth

\section{Personal Response}

Do you have any idea why the burden of preterm

II This is not understood, it is likely to be

What are you planning next for your research? I aim to understand how DAMP signals from the growing foetus can communicate towards the mother foetal membranes. 